\title{
Goal Programming Models and DSS for Manpower Planning of Airport Baggage Service
}

\author{
Sydney C.K. Chu ${ }^{1}$, Minyue Zhu ${ }^{2}$ and Liang Zhu ${ }^{3}$ \\ 1 Department of Mathematics, University of Hong Kong, Pokfulam Road, Hong \\ Kong, China. \\ schu@hku.hk \\ 2 Department of Mathematics, University of Hong Kong, Pokfulam Road, Hong \\ Kong, China. \\ zhuminyue@gmail.com \\ 3 Department of Mathematics, Fudan University, Shanghai, China. \\ godloveme_zhu@hotmail.com
}

Summary. Goal Programming (GP) models and Decision Support System (DSS) are two powerful tools dealing with manpower planning problems, not only on research level, but also as practical tools for industrial implementation. Goal programming is often useful as an optimization modeling technique for generating shift-duties of worker schedules. In our project for the baggage service agency at the Hong Kong International Airport, we proposed three model formulations based on the basic fixed-length shift duties generation model to approach various combinations of goals of manpower planning. Such an optimization modeling is built upon the essential foundation of a detailed data modeling and its analysis for all the driving parameters and demand/supply input necessary for numerical computations. The data model and GP model thus form the two integral components of the overall automation system - the DSS, which is an automatic computer based and user-friendly system to support management on planning decisions.

Key words: Manpower planning, gaol programming, decision support system.

\section{Introduction}

Goal Programming (GP) is often useful in duties generation problems (DGP) dealing with crew schedules (Chu, 2001) (Chu and Zhu, 2007). Many models (Azmat and Widmer, 2004)(Brusco and Jacobs, 2000)(Caprara et al., 2003) have been advocated in this area and work well when applied to practical projects (Chu, 2007). See for example, the reviews on methods, models and applications in (Burke and Petrovic, 2004)(Ernst et al., 2004). The decision support system (DSS), which realizes automation, brings mathematical optimization methods to a wide business use. This 
paper provides an optimization approach with its DSS, encompassing data modeling and GP modeling for a fixed-length shift-duties crew planning problem for Hong Kong Airport Services (HAS) Limited (Chu and Yuen, 2003). HAS is the primary handler of all ground services and support functions, including aircrafts, passengers and baggage at the Hong Kong International Airport.

Our project is to set the crew schedule for the baggage service agents BSA6 workers (being bus drivers for baggage) of HAS. When an airplane is due to arrive at the Airport, the control office of HAS Baggage Services will send BSA6 workers to the apron to pull back the baggage of the passengers to either the baggage handling basement level or "hot transfer" area in a number of trips. Similarly, when an airplane is ready to depart, all baggage must be sent on board. For operational purposes, the apron is considered as being divided into two zones by their distances to the basement. Airplanes are classified into two kinds: wide-body and narrow-body jets. There exist service standards of baggage services dealing with each kind of airplane for each zone, for example, the maximum time to finish offloading work, the least standby time for the last baggage for a departure flight and so on. Every BSA6 worker has a fixed-length regular duty of 9.5 hours of daily work inclusive of a one hour break preferable in the middle and possibly a limited length over-time duty immediately before or after the regular duty.

This paper consists of four further sections. In Section 2, we will provide detailed data models which generate demand data of work necessary as input for the proposed goal programming model. In Section 3, we state three versions of multi-objectives goal programming models as extended from the basic DGP-GP model (Chu, 2001) to this problem. Then, we provide in Section 4 some numerical results when we apply the models to the HAS problem instance, together with some analysis remarks. In Section 5, we introduce the decision support system developed for the HAS project as the final product. Some concluding remarks are given in the last section.

\section{Data Modeling}

Since the accurate estimation on data of demand is essential to the whole GP modeling approach, we put great efforts into the data modeling step to generate the data of demand as close as possible to the current situation while maintaining flexibility for future changes in flight schedules or transportation facilities. We present the overall demand pattern by a histogram (or bar-chart) of the number of required workers (or drivers in our problem here) over the operational time horizon of a day. This is done in two steps. First, we set up a system to estimate the individual demand for each flight on the flight schedule in the day separately; and secondly, we sum them up over half-hourly time intervals and make any necessary fine adjustments to ensure that the daily overall demand profile thus computed is practically realistic.

\subsection{Individual Demands}

Given the flight schedule, the first and most important thing to do is to estimate the number of trips needed for every flight, which is the foundation for all later calculations. Having the number the trips, we arrange the dispatching times for drivers which meet the HAS service standards. After reading a large number of past records 
and communicating with HAS supervisory staff, we pick out five major parameters which decide the number of trips and their trips' working times of the BSA 6 drivers. The five parameters in the descending order of importance are: $a_{1}(0,1)$-departure or arrival flight; $a_{2}(0,1)$-aircraft type; $a_{3}(0,1,2,3)$-airline carrier company; $a_{4}(0,1)$ multiple or single destination/origin city; $a_{5}(0,1,2,3,4,5)$-nature of the destination city for a departure flight / origin city for an arrival flight ("hot" or "normal" city with many or few flights). Each flight number gives input information for the values of $a_{1}, a_{2}, a_{3}, a_{4}$, and the destination/original city immediately; and these information together generate $a_{5}$. Then we get the number of trips of the flight $(f(a))$ according to these five parameters. The coefficients of the function are set based on the effect each parameter has, and on past experience and records about the number of trips. That is, the functional form of $f(a)$ is entirely derived from numerical "fitting". Here we give this function as follows:

$$
f(a)= \begin{cases}3 \times 1.1^{a_{4}} \times 1.0 & a_{5}=0,1 \\ 3 \times 1.1^{a_{4}} \times 1.9 & a_{5}=2,3 \\ 3 \times 1.1^{a_{4}} \times 2.0 & a_{5}=4 \\ 3 \times 1.1^{a_{4}} \times 1.6 & a_{5}=5\end{cases}
$$

This method of obtaining the number of trips performs quite well when validating the computational values with the recorded number of last year's operations and therefore we believe that it can provide reliable numbers dealing with new flights in the future. Having the number of trips of each flight, we set the operation times for every trip, satisfying the service standards under our plan. For example, the last trip to serve a flight will arrive at the basement within the permitted time standard.

\subsection{Overall Demands}

We divide one day into 48 time intervals, such that each interval has half an hour. For every single flight, with the results we get in the individual demand step, we obtain the number of trips over each time interval. And we sum up the number over each interval generated by every such flight and get the whole demand table for one day's schedule. After that, we make some ad hoc fine adjustments to the numbers to make the results closer to the real situation. For example, two nearby flight can sometimes share a trip in peak hours. We have two ways of data processing dealing with the peak hour data which are also adopted in the DSS. Further data value analysis enables us to omit eight time intervals (from 1:00 am to 4:59 am) with very little staff demand and put the first two mid-night intervals (from 00:00 am to 00:59 am) at the end of the preceding day due to the usual pattern of the airport working hours. Then we took the lower envelope and upper envelope of the numbers over each interval respectively to turn the computed fractional values during the whole process into integers. Hence, we have the final demands we need for the next GP models. Since the airport operates different weekly flight schedules for winter and summer, we work out totally 14 sets of daily data through the data modeling step. These are illustrated graphically in Figure 1, being Monday (Winter) demand. 


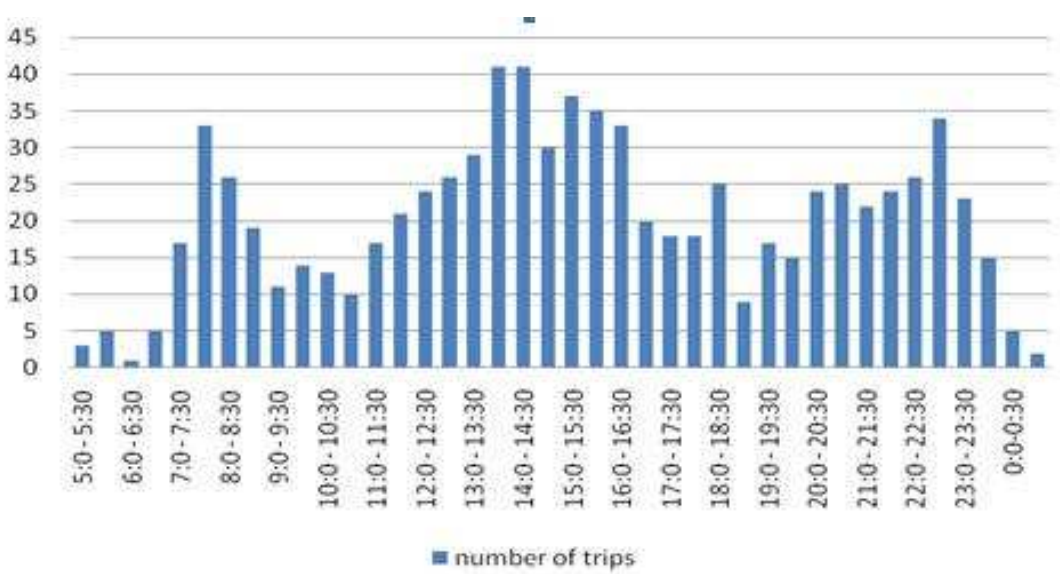

Fig. 1. Demand for Monday (Winter).

\section{Goal Program Modeling}

As its name implies, the Duty Generation Problem (DGP) model generates duties (performed by workers) in an optimal way to meet the known demand, over a contiguous number of time intervals. We describe below for completeness purpose its formulation relevant for our BAS6 problem setting here. A detailed account of original DGP formulations is given in an earlier paper (Chu, 2001) of one of the authors. In this project, we have several different goals: to control the maximum deviation of supply versus demand, to control the total number of regular workers required, and to control the total amount of over-time worker duties. We explore several model formulations all extended from the basic fixed-length shift-duties model to approach various combinations of goals.

\subsection{Model 1}

In model 1, we first add a large coefficient $W$ to the deviation variable that controls the highest number of over allocated workers in the working time interval over the day. Then this goal is combined with the goal of minimum total staff cost. Both regular duties and over-time duties are used and all demand is covered under this model.

We use the following notations. Let $J$ be the working time horizon, and $j=1 \ldots J$ index the individual hours. $R_{j}$ denotes the demand for interval $j$ and $d_{j}$ represents the over allocation (or over-achievement deviation variable in a goal programming context) at interval $j$. The length of a duty is denoted by $L$. The primary decision variable $x_{i k}$ is the number of allocated staffs that start regular duty from interval $i$ and breaks at the $k^{t h}$ interval after the start of duty, $k=1 \ldots L$. Hence for a working horizon of intervals $1 \ldots J$, we have for the index $i=S \ldots T$. The earliest start interval $S$ is such that $S \geq 1$; whereas the latest start interval $T$ is limited to $T \leq J-L+1$ (to finish work at interval $J$ ). Normally, $S=1$ as long as $R_{1}>0$ 
(there is demand for the very first interval); and $T=J-L+1$ whenever $R_{J}>0$ (there is demand for the very last interval). In principle the meal break could occur anywhere throughout the $L$ time intervals. In practice, there are two agreed times: the earliest start of break (ESB) and the latest start of break (LSB) times. This also requires another restriction on the break time index from $k=1 \ldots L$ to being $k=\mathrm{ESB}$ to LSB. For our case, the break time is required to be not earlier than 1 and also not later than 6 hours after the start of duty. With half-hour time intervals, this translates into $\mathrm{ESB}=3$ and $\mathrm{LSB}=14$. Another primary decision variable $y_{m n}$ is the number of allocated staffs who start their over-time duties at the $m^{\text {th }}$ interval and the duty lasts for $n$ intervals. Mathematically, the first model is given below.

The weighted goal function is:

$$
\operatorname{Min} W D+\sum_{i=S}^{T} \sum_{k=E S B}^{L S B} c_{k} x_{i k}+g \times \sum_{m=L+1}^{J-n+1} \sum_{n} n y_{m n}
$$

The constraints are:

$$
\begin{aligned}
& \sum_{\substack { i=p \\
\begin{subarray}{c}{k \neq j-i \\
k \neq j-i+1{ i = p \\
\begin{subarray} { c } { k \neq j - i \\
k \neq j - i + 1 } }\end{subarray}}^{q} \sum_{i k}^{L S B}+\sum_{m=j-n+1}^{j} \sum_{n} y_{m n}-d_{j}=R_{j}, \quad j=1 \ldots J \\
& \sum_{n} y_{i+L, n} \leq \sum_{k} x_{i, k}, \quad i=1 \ldots I \\
& d_{j} \leq D, \quad j=1 \ldots J
\end{aligned}
$$

Here $p=\max \{j-L+1, S\}, q=\min \{j, T\}$, and $x_{i k}, y_{m n}, d_{j}$ are non-negative variables.

We see that the LHS of the first constraint is the total work contribution as a function of staff duties containing both regular and over-time duties. The coefficients $c_{k}$ represent the usual unit regular pay rates according to the break hour. $g$ is the pay ratio of one hour over-time pay over one day regular pay. The second constraint ensures that the over-time duties can only be performed immediately before or after the regular duties. The single variable $D$ of the last constraint records the maximum (over achievement) deviation over all time intervals, with weighting parameter $W$.

\subsection{Model 2}

In model 2, we set up realistic caps to the total number of regular workers or overtime duties to get practically achievable planning results under different manpower requirement controls. All demand is covered under model 2. The goal function is the same as in model 1 , but we add either or both of the following two constraints:

$$
\begin{gathered}
\sum_{i} \sum_{k} x_{i k} \leq c_{0} \\
\sum_{m} \sum_{n} y_{m n} \leq c_{1}
\end{gathered}
$$

Here $c_{0}$ and $c_{1}$ are two user-specified constants. We should notice that if we add a cap to the total over-time duties, we can always get feasible solutions since over-time 
duties can be replaced by regular duties anyway. But if we add a cap to the total regular duties and the cap is too low, we sometimes cannot get feasible solutions. This reveals that the over-time duties are not as powerful as they seem to be. Using the over-time duties are actually only extending the length of duties of workers. In our project in HAS, $c_{0}$ and $c_{1}$ are set slightly less than the existing numbers of regular and over-time duties, respectively.

\subsection{Model 3}

In the third model, we set realistic caps to the number of total regular and overtime duties and we use a new kind of staffs - the part-time workers. The goals are approached in two steps. In the first step, we set caps to the number of total regular and over-time duties and we allow shortage of manpower, which means that not all the demand is covered in step one. And we control the maximum shortage. The system is given as follows:

$$
\text { Min Maxe }
$$

The constraints are:

$$
\begin{aligned}
& \sum_{i=p}^{q} \sum_{\substack{k \neq j-i \\
k \neq j-i+1}}^{L S B} x_{i k}+\sum_{m=j-n+1}^{j} \sum_{n} y_{m n}+e_{j} \geq R_{j}, j=1 \ldots J \\
& e_{j} \leq \text { Maxe }, \quad j=1 \ldots J \\
& \sum_{i} \sum_{k} x_{i k} \leq c_{0}, \quad \sum_{m} \sum_{n} y_{m n} \leq c_{1}
\end{aligned}
$$

Here, $e_{j}$ indicates the shortage of manpower in the interval $j$, and Maxe is the maximum shortage among all the operating intervals of the day.

In the second step, we fill up any such shortage $e_{j}$ by part-time duties in anticipation of the part-time workers to be subsequently made available. The part-time workers are assumed to be of totally flexible work duration. Thus, in this way, all the demand is also covered under model 3. In this step, the goal function is the same as in model 1 :

$$
\operatorname{Min} W D+\sum_{i=S}^{T} \sum_{k=E S B}^{L S B} c_{k} x_{i k}+g \times \sum_{m=L+1}^{J-n+1} \sum_{n} n y_{m n}
$$

The constraints are:

$$
\begin{aligned}
& \sum_{i=p}^{q} \sum_{\substack{k \neq j-i \\
k \neq j-i+1}}^{L S B} x_{i k}+\sum_{m=j-n+1}^{j} \sum_{n} y_{m n}-d_{j}+e_{j}=R_{j}, \quad j=1 \ldots J \\
& \sum_{n} y_{i+L, n} \leq \sum_{k} x_{i, k}, \quad i=1 \ldots I \\
& d_{j} \leq D, \quad e_{j} \leq c_{2}, \quad j=1 \ldots J
\end{aligned}
$$

Here, $c_{2}$ is the computed result from step one. 


\section{Numerical Results and Comments}

\subsection{Numerical Results}

Here we provide some numerical results when applying the model to our problem. For our problem instance, the number of working hours of a day is 20 , which means $J=40$. The length of duty is 9.5 hours, which means $L=19$. The break is restricted to $\mathrm{ESB}=3$ and $\mathrm{LSB}=14$, as mentioned before. We prefer staff if possible taking break close to the middle of the duty, which means $c_{k}$ is assigned a smaller value when $k$ is closer to the middle between 3 and 14. $g=0.092 . n=2,3,4,5,6$, which means that the over-time duties can last 1 to 3 hours. Having all these, we code the model in LINGO (Schrage, 2005) to compute the solution to the optimization problem and we plot the results in chart form and show the detailed values in table form. They are illustrated as follows:

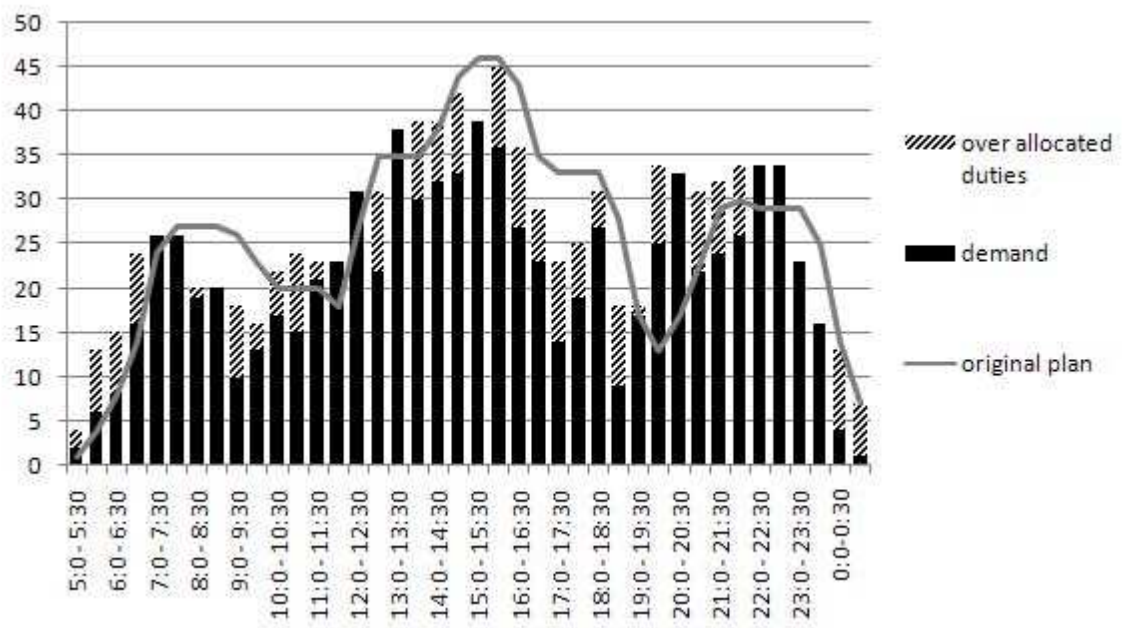

Fig. 2. Schedule for Sunday (Summer, Model 1).

In Figure 2, the dark bars represent the demand for the time intervals and the latticed bars represent the over allocated duties. Together, an allocation is plotted by the stacked dark and latticed bars. The line graph is the original plan used by the airport.

In Table 1, the first four rows are related to regular duties and the last row refers to over-time duties. For example, $(530,2.5) 6$ means that there are 6 allocated workers who start their regular duties at 5:30 and take the break 2.5 hours after the start. We should notice that the over-time duty is immediately after the regular duty, which can also be seen in this table. For example, the 5 duties starting at 21:30 and lasting for 1.5 hours are therefore actually after their regular duties starting at 12:00. Likewise, the 7 duties starting at 22:30 and lasting for 1 hour are after the $(1+6=) 7$ duties starting at 13:00. 
Table 1. Schedule for Sunday (Summer, Model 1).

\begin{tabular}{|l|l|l|l|}
\hline$(500,4) 4$ & $(530,2.5) 6$ & $(530,5.5) 3$ & $(600,4) 2$ \\
\hline$(630,3.5) 4$ & $(630,7) 5$ & $(700,2.5) 2$ & $(1200,4.5) 5$ \\
\hline$(1300,1.5) 1$ & $(1300,5.5) 6$ & $(1330,5) 3$ & $(1330,6) 3$ \\
\hline$(1430,5.5) 1$ & $(1430,6) 2$ & $(1500,2) 6$ & $(1530,3) 7$ \\
\hline & & & \\
\hline$(2130,1.5) 5$ & $(2230,1) 7$ & & \\
\hline
\end{tabular}

\subsection{Analytical Comments and Comparison of the Models}

By analyzing the data and the computed results, we find that the demand patterns are similar among the seven days of a week. Each day, there are 3 or 4 peaks at around 7:30, 13:00, 15:00 and 22:30, respectively. In fact, these are the actual peak hours at the airport. Under the original plan now in use by the airport, most of the tasks can be completed according to standards except for some peak hours, especially in the morning and in the late evening. Many workers, however, can be found idle in most of the other working hours. This situation is reflected in Figure 2. The main objective achieved by our project is to generate the daily crew scheduling plan which ensures that all tasks can be completed with minimum human resources. It should not be used as the detailed roll list by the duty manager since flights may delay or duties can be completed faster or slower than estimated. It should be treated as support for decision making on the daily planning, and for total number of staffs in the long run.

Comparison is made among results from Model 1 and Model 3, as shown in Table 2. Here, $\mathrm{X}$ is the number of regular workers, $\mathrm{Y}$ is the number of over-time workers and $\mathrm{Z}$ is the number of part-time workers (with a part-time duty lasting for 4 hours).

Table 2. Comparison of Model 1 and Model 3.

\begin{tabular}{|c|l|l|l|l|l|l|l|}
\hline & \multicolumn{3}{|c|}{ Model 1 } & \multicolumn{3}{c|}{ Model 3 } & \multicolumn{2}{c|}{ Standard } \\
\hline Day & $\mathrm{X}$ & $\mathrm{Y}$ & $\mathrm{X}$ & $\mathrm{Y}$ & $\mathrm{Z}$ & $\mathrm{X}$ & $\mathrm{Y}$ \\
\hline Mon & 61 & 20 & 60 & 9 & 7 & 60 & 15 \\
\hline Tue & 56 & 12 & 55 & 8 & 2 & 57 & 10 \\
\hline Wed & 59 & 14 & 57 & 11 & 1 & 58 & 15 \\
\hline Thu & 59 & 15 & 56 & 12 & 4 & 58 & 15 \\
\hline Fri & 63 & 11 & 59 & 12 & 3 & 60 & 14 \\
\hline Say & 58 & 16 & 56 & 15 & 3 & 58 & 15 \\
\hline Sun & 60 & 12 & 58 & 14 & 3 & 59 & 14 \\
\hline \hline Total & 416 & 100 & 401 & 81 & 23 & 410 & 98 \\
\hline
\end{tabular}

When model 3 is used, the $\mathrm{X}$ and $\mathrm{Y}$ can be controlled under the operating standards. Here "Standard" in the table refers to "operating standard", or actually the 
current practices, which do not guarantee full demand coverage (at all hours). The numbers of part-time workers are not significant except for Monday. This indicates that the officials of HAS could consider adding a new mode of part-time duty to the manpower staffing with a reasonable pay.

\section{Decision Support System}

Since the GP model solves the core problem of manpower planning - given the demand and optimization goals, it generates the manpower supply schedule forming the essential part of an integrating decision support system (DSS) towards actual implementation. In this project of baggage service agents (workers) of the Hong Kong International Airport, our DSS tool is designed as a computer and knowledge based information system to support user-friendly decision making activities. It is developed as a PC-based system to generate crew schedules automatically giving the input information of flights schedule and their supportive operational parameters. When the clients input or import the flights schedule, the data model system component builds up the daily demand profile (over individual half-hourly time intervals) of workers automatically, which is also the input data for the next GP model. Since the optimization GP system component is built upon the essential foundation of such a detailed data modeling characterized by its analysis for all the driving parameters and demand/supply input necessary for numerical computations, data model and GP model thus form the two integral components of the overall automation system, and finally the DSS. Here in Figure 3 is an illustrative screen capture of the main input page of the DSS.

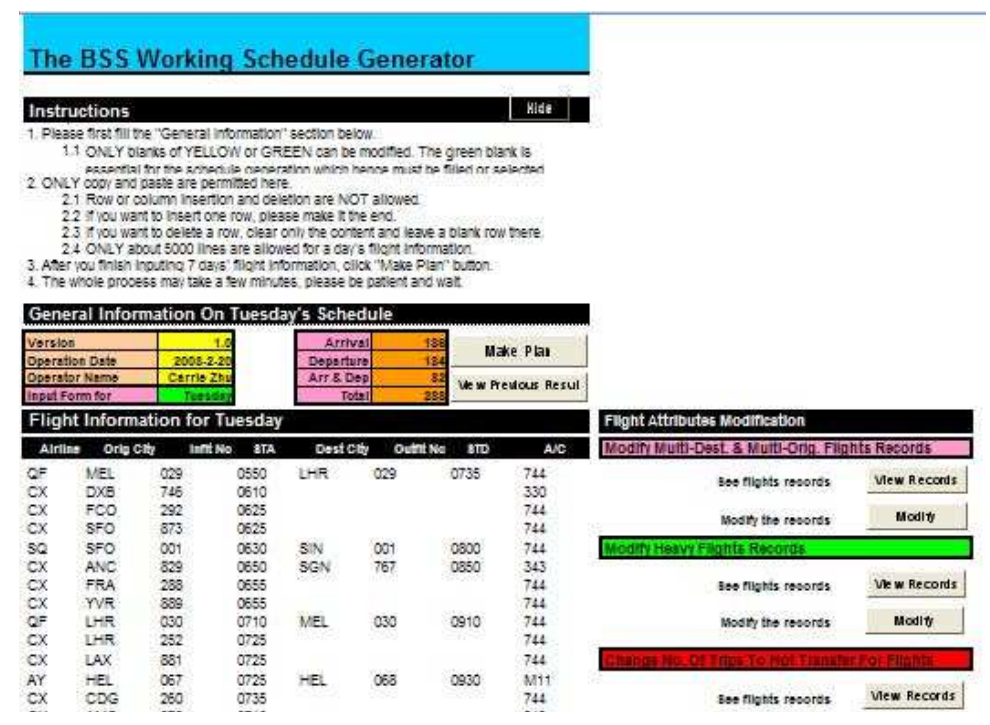

Fig. 3. Main input page of the DSS. 
User can input or paste the flights schedule to the input area and simply click the "Make Plan" button to get the detailed and overall schedule in about 25 seconds. The user-friendly spreadsheet based interface also provides the ultimate flexibility for users to make changes to the original characteristics of problem information, and to perform what-if analysis, on top of its generic calculations of the manpower planning function.

\section{Concluding Remarks}

Goal Programming (GP) models and Decision Supporting System (DSS) are used as practical tools for implementation in our project dealing with manpower planning problems of HAS at the Hong Kong International Airport. The overall automation DSS has been designed with an view of being wholely computer based and userfriendly to support management uses for longer-term planning decisions, rather than real-time dispatching actions.

The building and using of the system have since the start of the project been a joint effort with the decision makers, the senior management of the Baggage Services Section of HAS. Detailed flight and workload data were provided and discussed at working meetings at the HAS Airport office. Our central GP modeling idea of duties generation to cover required workload was also explained and understood. The reaction to our methodology and the opinions on our model findings were rather favourable. This was partly due to the fact that our numerical results tallied well with their expectation on possible operational improvement they were looking and hoping for. The main benefit as we could ascertain later was mainly in terms of their improved future planning on the staffing level and the ease of the DSS uses for answering what-if type staffing questions in general.

Acknowledgement. This work is partially supported by the Hong Kong RGC Competitive Earmarked Research Grant (CERG) Award: HKU 7126/05E.

\section{References}

Azmat, C. S. and Widmer, M. (2004). A case study of single shift planning and scheduling under annualized hours: A simple three-step approach. European Journal of Operational Research, 153, 148-175.

Brusco, M. J. and Jacobs, L. W. (2000). Optimal models for meal-break and starttime flexibility in continuous tour scheduling. Management Science, 46, 16301641.

Burke, E. and Petrovic, S. (2004). Timetabling and rostering. European Journal of Operational Research, 153, 1-2.

Caprara, A., Monaci, M., and Toth, P. (2003). Models and algorithms for a staff scheduling problem. Mahtematical Programming, 89, 445-476.

Chu, S. C. K. (2001). A goal programming model for crew duties generation. Journal of Multi-criteria Decision Analysis, 10, 143-151. 
Chu, S. C. K. (2007). Generating, scheduling and rostering of shift crew-duties: Applications at the hong kong international airport. European Journal of Operational Research, 177, 1764-1778.

Chu, S. C. K. and Yuen, C. S. (2003). Planning and scheduling staff duties by goal programming. In T. Tanaka and M. Inuiguchi, editors, Multi-Objective Programming and Goal-Programming, pages 309-316. Advances in Soft Computing, Springer-Verlag Berlin Heidelberg.

Chu, S. C. K. and Zhu, M. (2007). Data and optimaization modeling for manpower planning of an airport baggage service. In Proceedings of the Institute of Industrial Engineers Annual Conference -2007 Industrial Engineering Research Conference (IERC 2007), Nashvill, TN, USA, 19-23 May 2007, pages 19-23.

Ernst, A. T., Jiang, H., Krishnamoorthy, M., and Sier, D. (2004). Staff scheduling and rostering: A review of applications, methods and models. European Journal of Operational Research, 153, 3-27.

Schrage, L. (2005). Optimization Modeling with LINGO. Lindo Systems Inc., Chicago, 5th edition. 